\title{
Residência em Medicina de Família e Comunidade para a formação de recursos humanos: o que pensam gestores municipais?
}

\author{
Family and Community Medicine Residency Programs for training \\ the health workforce: what do municipal health managers think?
}

\author{
Ana Paula Tussi Leite (https://orcid.org/0000-0002-1797-195X) ${ }^{1}$ \\ Isabel Brandão Correia (https://orcid.org/0000-0002-6950-8684) ${ }^{2}$ \\ Patrícia Sampaio Chueiri (https://orcid.org/0000-0002-0811-3910) ${ }^{3}$ \\ Thiago Dias Sarti (https://orcid.org/0000-0002-1545-6276) ${ }^{4}$ \\ Adelson Guaraci Jantsch (https://orcid.org/0000-0002-3012-5619) ${ }^{5}$ \\ Alice Paul Waquil (https://orcid.org/0000-0002-4959-1433) ${ }^{1}$ \\ Scheila Mai (https://orcid.org/0000-0003-1800-0140) ${ }^{1}$ \\ Vitória Silva da Rosa (https://orcid.org/0000-0001-9105-559X) ${ }^{1}$ \\ Elvira Alicia Aparicio Cordero (https://orcid.org/0000-0003-4193-1939) ${ }^{1}$ \\ Daniel Knupp Augusto (https://orcid.org/0000-0002-5968-4932) ${ }^{6}$ \\ André Ferreira Lopes (https://orcid.org/0000-0003-3503-3614) ${ }^{7}$ \\ Maria Eugênia Bresolin Pinto (https://orcid.org/0000-0002-7536-6299) ${ }^{1}$
}

${ }^{1}$ Programa de Apoio ao Desenvolvimento Institucional do Sistema Único de Saúde (PROADISUS), Hospital Moinhos de Vento. R. Ramiro Barcelos 910, Moinhos de Vento. 90035-000 Porto Alegre RS Brasil.aptussi@gmail.com ${ }^{2}$ Faculdade de Ciências Médicas, Universidade de Pernambuco. Recife PE Brasil.

${ }^{3}$ Curso de Medicina, Faculdade Israelita de Ciências da Saúde. São Paulo SP Brasil. ${ }^{4}$ Programa de PósGraduação em Saúde Coletiva, Centro de Ciências da Saúde, Universidade Federal do Espírito Santo. Vitória ES Brasil. ${ }^{5}$ Programa de PósGraduação em Saúde Pública, Instituto de Medicina Social, Universidade do Estado do Rio de Janeiro. Rio de Janeiro RJ Brasil.

${ }^{6}$ Unimed Belo Horizonte. Belo Horizonte MG Brasil. ${ }^{7}$ Secretaria Municipal de Saúde do Rio de Janeiro. Rio de Janeiro RJ Brasil.

\begin{abstract}
The training, recruitment and retention of primary care professionals is a constant challenge in Brazil. The recent expansion of family and community medicine residency programs in the country coexists with gaps in the literature on the effects of this process. This article explores municipal health managers' understanding of these programs and the role they play in professional training and improving the quality of health care. We conducted a quantitative and qualitative analysis of the responses to questionnaires answered by 48 health managers working in municipal health services affiliated to residency programs. A descriptive statistical analysis of the quantitative data was performed and the qualitative data were analyzed using thematic analysis. The findings show that efforts were made to incorporate family and community doctors into the health care network and that managers recognized the potential residency program have to improve the quality of care and enhance professional training. Weaknesses were found in actions to improve infrastructure and facilities and the organization of the services affiliated to the programs. This study highlights the potential of residency programs for addressing longstanding problems in primary health care in Brazil when combined with actions to strengthen services, human resources and the programs.

Key words Primary Health Care, Family Practice, Internship and Residency
\end{abstract}

Resumo Treinamento especializado, provimento e fixação de profissionais na Atenção Primária à Saúde são desafios prementes no Brasil. A recente expansão dos Programas de Residência em Medicina de Família e Comunidade coexiste com lacunas na literatura sobre o efeito deste processo. O objetivo do trabalho é explorar a perspectiva do gestor municipal de saúde acerca das estratégias para o fortalecimento das residências e do papel destas na formação profissional e na qualificação da atenção. Trata-se de um estudo de análise quantitativa e qualitativa, com aplicação de questionário a gestores de municípios cenários destes programas. As respostas de 48 sujeitos foram submetidas a estatística descritiva e análise de conteúdo. Os resultados revelam um esforço em incorporar o Médico de Família e Comunidade na rede de atenção à saúde, uma percepção do potencial das residências no incremento da qualidade da atenção e da formação profissional e fragilidades nas ações para melhoria da estrutura e organização dos serviços com residentes. Vislumbra-se assim o potencial das residências para a superação de problemas históricos da Atenção Primária à Saúde brasileira, se atrelada a ações de fortalecimento do serviço, dos recursos humanos e do próprio programa.

Palavras-chave Atenção Primária à Saúde, Medicina de Família e Comunidade, Internato e Residência 


\section{Introdução}

Os avanços ocorridos nos últimos 40 anos ao redor do mundo na expansão e melhoria da Atenção Primária à Saúde (APS) coexistem com uma ampla variedade de carências estruturais que ainda ameaçam o desenvolvimento social dos países de baixa e média renda ${ }^{1,2}$. Dentre estas, destaca-se a formação de recursos humanos para a saúde como um dos principais problemas a ser enfrentado ${ }^{3,4}$.

Mesmo com evidências robustas de que uma APS forte e estruturada torna todo o sistema de saúde mais eficiente, produz melhores resultados de saúde e cuidados mais equânimes ${ }^{5}$, o treinamento especializado para o cuidado em saúde, junto com o provimento e a fixação de profissionais, são desafios que ainda estão longe de serem sanados no Brasil.

Esse desafio histórico já foi objeto de intervenção por meio de políticas de saúde e educação de variados governos em diferentes níveis federativos do país. Nos últimos 15 anos, os programas PET-Saúde ${ }^{6}$, a Universidade Aberta do SUS (UNASUS $)^{7}$, o Programa de Apoio à Formação de Médicos Especialistas em Âreas Estratégicas (Pró -Residência $)^{8}$, o Programa de Valorização da Atenção Básica (PROVAB) ${ }^{9}$, o Programa Mais Médicos $(\mathrm{PMM})^{10} \mathrm{e}$, mais recentemente, o Programa Médicos pelo Brasil ${ }^{11}$, foram iniciativas federais que buscaram intervir nos problemas relacionados à formação, ao provimento e a fixação de médicos em áreas remotas do país, ao mesmo tempo em que se propunham a garantir maior efetividade do cuidado através de incentivos ao treinamento profissional.

A carência estrutural da formação e do provimento profissional não é um problema exclusivo da APS, mas sim reflexo da constituição política e econômica do país que resulta em profundas desigualdades sociais, seja entre suas distintas regiões geográficas, seja entre metrópoles e zonas rurais ${ }^{12}$. Tais processos históricos geradores de inequidades institucionais se expressam fortemente em todo o aparato de formação médica brasileira, tanto na graduação quanto na formação especializada, por meio de Residência Médica (RM). As escolas médicas e os Programas de Residência Médica (PRM) são distribuídos de maneira desigual no território nacional, concentrando-se em grandes centros urbanos e nas regiões Sul e Sudeste ${ }^{13,14}$.

Considerando que (1) no Brasil a especialização médica através da RM não é obrigatória para a prática da profissão, como ocorre em grande parte dos países de alta renda ${ }^{15}$; (2) que este nível de formação não possui estrutura suficiente para a ab- sorção de todos os egressos das escolas médicas do país ${ }^{14}$; (3) que, por estes motivos, parte significativa dos médicos optam por não se especializarem ou por adquirir o título de especialista por outras vias legais autorizadas no país ${ }^{14,15}$; e (4) que a RM é o formato de formação profissional mais adequado para a garantia da qualidade da prática profissional e dos cuidados prestados à população $0^{16}$, depreende-se que tais problemas podem resultar em desigualdades em saúde que se somam e agravam aos problemas sociais e econômicos do país.

Esta realidade é ainda mais evidente na APS. Comparado a outras experiências internacionais, a proporção de vagas de RM em MFC no Brasil é pequena em relação ao total de vagas ofertadas e a sua ocupação é de cerca de apenas 30\% nos últimos dois anos ${ }^{17}$. O número de especialistas em Medicina de Família e Comunidade (MFC) no país é insuficiente para suprir a necessidade do Sistema Único de Saúde (SUS), e o atual quantitativo de MFCs formados anualmente, apesar do expressivo crescimento nos últimos anos em decorrência das políticas indutoras vinculadas ao PMM e de experiências municipais, torna distante o horizonte de resolução do problema ${ }^{14,18}$.

Como citado acima, uma das principais inovações na formação do MFC no Brasil é a implementação de PRMFC robustos vinculados às gestões municipais de saúde. Tais PRMFC ofertam grande número de vagas anuais; implementam dispositivos incomuns de gestão no quadro nacional atual, como complementações nos valores das bolsas dos médicos residentes; alcançam boas taxas de ocupação de suas vagas de RM; e por isso contribuem para a provisão local de profissionais médicos qualificados para a APS. Dentre tais experiências, destacam-se Rio de Janeiro, Curitiba, Florianópolis, São Bernardo do Campo, Palmas, Recife e João Pessoa ${ }^{19-21}$.

A consolidação de políticas públicas eficientes que garantam uma força de trabalho qualificada e em quantidade suficiente para a consolidação da APS está atrelada a uma compreensão dos facilitadores e das barreiras neste processo. Investigar a compreensão de gestores municipais, enquanto elaboradores de políticas públicas e tomadores de decisão, sobre as residências em MFC como uma ferramenta para a formação de recursos humanos para a APS brasileira pode ajudar a esclarecer quais os motivos que mobilizam e desmotivam esses atores a investirem neste tipo de formação no Brasil, principalmente ao se considerar a escassez de literatura a respeito ${ }^{22,23}$.

Tendo em vista que a imensa maioria dos PRMFC realizam suas atividades em serviços 
municipais de $\mathrm{APS}^{19}$, que parte significativa das políticas de provimento e expansão dos PRMFC implementadas nos últimos anos tem seu foco no município ${ }^{24} \mathrm{e}$ que são as perspectivas dos gestores dos sistemas municipais de saúde que orientam as políticas locais, incluindo as de formação profissional, este artigo tem como objetivo explorar a compreensão, as ações desenvolvidas e as atitudes dos gestores municipais acerca da residência em Medicina de Família e Comunidade como uma estratégia para formação de recursos humanos para a APS brasileira.

\section{Métodos}

Este artigo é recorte de uma pesquisa de abrangência nacional intitulada "Caracterização dos Programas de Residência em Medicina de Família e Comunidade no Brasil", executada pelo Hospital Moinhos de Vento, por meio do Programa de Apoio ao Desenvolvimento Institucional do Sistema Único de Saúde (PROADI-SUS). Ela é caracterizada por ser um estudo de métodos mistos e este artigo trabalha especificamente dados relacionados aos gestores municipais através de análises quantitativas e qualitativas que serão detalhadas a seguir.

Os PRMFC e municípios incluídos nesta pesquisa foram identificados a partir de dados fornecidos pelo Ministério da Saúde e obtidos na base da Comissão Nacional de Residência Médica. Uma equipe de pesquisadores revisou os dados para identificação de inconsistências, duplicações e omissões, delimitando a população da pesquisa a gestores municipais, supervisores, preceptores, médicos residentes e egressos relacionados aos 249 PRMFC identificados como ativos no ano de 2019, distribuídos em 157 municípios do país. Os sujeitos que compõem a população deste estudo são exclusivamente os gestores de municípios cujos sistemas de saúde são cenários de prática de PRMFC.

Questionários online, específicos para cada um dos grupos investigados, foram utilizados como instrumentos de coleta de dados. Estes questionários foram desenvolvidos de forma a obter um amplo leque de informações sobre a estruturação, gestão e execução dos PRMFC do país, traçando um perfil destes a partir de múltiplos olhares e percepções. Uma equipe multiprofissional e com expertise diversa em métodos de pesquisa desenvolveu uma primeira versão destes questionários, que foram então submetidos a duas fases de análise, ajustes e testagem. Num primeiro momento, especialistas em MFC selecionados pela Sociedade Brasileira de Medicina de Família e Comunidade (SBMFC) e técnicos vinculados ao Ministério da Saúde analisaram os instrumentos, sendo feitas alterações, exclusões ou inclusões de itens. Em seguida, um novo grupo de especialistas em MFC respondeu os questionários, sendo analisados reprodutibilidade e tempo de resposta. Novos ajustes foram realizados e chegou-se à versão final dos instrumentos para coleta de dados.

Especificamente, o questionário voltado aos gestores municipais explora dados sociodemográficos, da estrutura dos serviços de saúde e instituições de ensino onde funcionam os PRMFC, do processo de integração ensino-serviço e das avaliações que os sujeitos fazem da especialidade MFC e do PRMFC. Ao todo foram 30 questões, que quando desdobradas geraram 72 variáveis, sendo 50 quantitativas e 22 qualitativas. A natureza das questões foi diversificada, e incluiu os tipos múltipla escolha, checkbox, respostas curtas e dissertativas, para garantir que os sujeitos não apenas respondessem de forma guiada às temáticas abordadas no instrumento, como também expressassem de forma narrada suas percepções gerais.

A coleta de dados de todas as populações estudadas ocorreu entre janeiro e abril de 2020. Os potenciais participantes da pesquisa foram acessados através de telefone, e-mail, aplicativos de mensagens e plataformas digitais, para apresentação dos objetivos e o método de pesquisa e posterior envio de links específicos que davam acesso aos instrumentos via plataforma online Research Electronic Data Capture (REDCap). Especificamente para a coleta de dados do gestor municipal, com o intuito de identificar o sujeito mais intrinsecamente vinculado aos PRMFC, as Secretarias Municipais de Saúde (SMS) dos municípios selecionados foram contactadas para apresentação do projeto e para indicação do sujeito reconhecidamente mais apropriado a participar do estudo. Para sensibilizar os sujeitos sobre a importância do projeto e como estratégia para aumento da taxa de resposta, a pesquisa foi amplamente divulgada em redes sociais, incluindo perfis e canais de comunicação voltados para os MFC, e sítio do Conselho Nacional de Secretarias Municipais de Saúde (CONASEMS).

O banco de dados da pesquisa foi gerenciado por uma pesquisadora expert neste procedimento. A análise quantitativa se deu através de estatística descritiva simples com frequências absolutas e relativas realizadas através do software 
estatístico R (versão 3.6.1). Os dados qualitativos foram submetidos à análise de conteúdo temática $^{25}$, sendo que o corpus textual foi composto pelas respostas dos sujeitos às questões abertas dispostas no instrumento, sendo submetido a leitura exaustiva, codificação inicial e enucleação de sentidos com a definição dos núcleos temáticos, sendo apresentados neste artigo os mais relacionados aos objetivos deste. Buscou-se então realizar análises e reflexões integradas dos dados quantitativos e qualitativos pelo grupo de pesquisadores responsáveis pela pesquisa em sessões coletivas de discussão dos resultados.

Os sujeitos da pesquisa foram incluídos no estudo após sua aprovação dos procedimentos e assinatura do Termo de Consentimento Livre e Esclarecido. Todas as normas éticas dispostas na Lei 466/2012 foram seguidas e o projeto foi aprovado pelo Comitê de Ética em Pesquisa do Hospital Moinhos de Vento.

\section{Resultados e discussão}

Os resultados e suas análises serão apresentados em três eixos. Inicialmente serão caracterizados os sujeitos da pesquisa e os municípios aos quais estão vinculados. Em seguida, serão expostas as estratégias de gestão adotadas pelos municípios para manutenção e ampliação dos PRMFC. E, por fim, serão apresentados três núcleos temáticos resultantes da análise qualitativa: efeitos da residência médica no sistema e serviços de saúde; efeitos da residência médica na formação profissional; percepções dos gestores sobre o médico residente e o PRMFC.

\section{Caracterização dos sujeitos da pesquisa}

Ao todo, 48 gestores de municípios responderam o questionário, o que representa 30,6\% do total de municípios que são cenários para os PRMFC no país (Tabela 1 ). São em sua maioria pessoas do sexo feminino $(n=30 ; 62,5 \%)$, brancas $(\mathrm{n}=30 ; 62,5 \%)$, com até 50 anos de idade $(\mathrm{n}=36$; $76,7 \%)$, com ensino superior completo $(n=48$; $100,0 \%)$, residentes nas regiões sul e sudeste $(\mathrm{n}=33 ; 68,8 \%)$. Grande parte possui graduação na área da saúde $(\mathrm{n}=38 ; 80,9 \%)$, sendo medicina e enfermagem as principais formações nesta área $(n=28 ; 73,6 \%)$. Este perfil é semelhante ao encontrado na Pesquisa Nacional dos Secretários Municipais de Saúde ${ }^{26}$.

Os entrevistados eram, na maioria, gestores da APS e da Educação em Saúde do município $(n=25 ; 53,2 \%)$ e exerciam a função por 3 anos ou menos ( $n=30 ; 62,5 \%)$ (Tabela 1$)$. Os sujeitos que ocupam cargos intermediários na gestão do sistema de saúde municipal, por um curto período de tempo, ocupam a função de tomadores de decisão sobre estratégias de fixação e provimento, o que pode explicar a variabilidade e instabilidade da consolidação dessas iniciativas ${ }^{19}$.

\section{Caracterização dos municípios quanto ao ensino}

A maior parte dos municípios da pesquisa é cenário de prática para instituições de ensino com graduação em medicina $(n=37 ; 78,7 \%)$, principalmente de natureza privada $(n=20 ; 54,0 \%)$; e também é palco para apenas um PRMFC $(n=27$; $56,3 \%$ ), havendo municípios com até 7 programas em funcionamento. O perfil mais comum na amostra é de municípios com 1 PRMFC funcionando em até 4 unidades básicas de saúde $(n=18$; 37,5\%). O total de Unidades Básicas de Saúde (UBS) dos municípios que participam da pesquisa é de 2502. Considerando que ao todo 390 são ocupadas com residentes, elas correspondem a $15,6 \%$ do total de UBS desses municípios ${ }^{27}$. Esse percentual é um pouco maior do que o correspondente de especialistas em MFC que atuam em UBS no Brasil. Quanto às escolas privadas, esse percentual é condizente com a realidade do país, no qual $61 \%$ das escolas médicas são instituições privadas $^{28}$. Por outro lado, independente da natureza da instituição, dentre os gestores que relataram a existência de graduação de medicina no município, a maioria $(n=30 ; 63,8 \%)$ mencionou a complementação com bolsa financeira para os residentes e a APS como campo de prática para a graduação como as principais estratégias da gestão para fomentar a procura dos estudantes pelas vagas de residência. (Tabela 2). A estratégia de aproximação dos estudantes com os cenários de prática das residências em MFC é reconhecida como catalisadora da escolha pela especialidade, revelando que os gestores que a viabilizam podem estar num caminho de círculo virtuoso para o fortalecimento da especialidade ${ }^{29}$.

\section{Estratégias da gestão municipal para os PRMFC}

No que diz respeito a participação da gestão no planejamento da abertura dos PRMFC que não são próprios do município, quase metade $(n=22 ; 45,8 \%)$ dos gestores afirmaram que a Secretaria Municipal de Saúde teve participação 
Tabela 1. Características sociodemográficas e profissionais dos gestores, Brasil, 2020.

\begin{tabular}{|c|c|}
\hline Variável & n (\%) \\
\hline \multicolumn{2}{|l|}{ Faixa etária $(n=47)$} \\
\hline Até 29 anos & $2(4,3)$ \\
\hline $30-39$ & $17(36,2)$ \\
\hline $40-49$ & $17(36,2)$ \\
\hline 50 anos ou mais & $11(23,4)$ \\
\hline \multicolumn{2}{|l|}{ Sexo $(n=48)$} \\
\hline Feminino & $30(62,5)$ \\
\hline Masculino & $18(37,5)$ \\
\hline \multicolumn{2}{|l|}{ Raça/Cor $(n=48)$} \\
\hline Preta & $2(4,2)$ \\
\hline Parda & $14(29,2)$ \\
\hline Branca & $30(62,5)$ \\
\hline Amarela & $1(2,1)$ \\
\hline Não declarada & $1(2,1)$ \\
\hline \multicolumn{2}{|l|}{ Escolaridade $(\mathrm{n}=48)$} \\
\hline Ensino superior completo & $48(100,0)$ \\
\hline \multicolumn{2}{|l|}{ Área de graduação $(\mathrm{n}=47)$} \\
\hline Saúde & $38(80,9)$ \\
\hline Outras & $9(19,1)$ \\
\hline \multicolumn{2}{|l|}{$\begin{array}{l}\text { Categoria profissional na área da saúde } \\
(\mathrm{n}=38)\end{array}$} \\
\hline Medicina & $14(36,8)$ \\
\hline Enfermagem & $14(36,8)$ \\
\hline Outras & $10(26,4)$ \\
\hline \multicolumn{2}{|l|}{ Região do país ( $\mathrm{n}=48$ ) } \\
\hline Norte & $3(6,3)$ \\
\hline Nordeste & $10(20,8)$ \\
\hline Centro-Oeste & $2(4,2)$ \\
\hline Sudeste & $18(37,5)$ \\
\hline Sul & $15(31,3)$ \\
\hline \multicolumn{2}{|l|}{ Função no município $(\mathrm{n}=47)$} \\
\hline Secretário(a) Municipal de Saúde & $8(17,0)$ \\
\hline Gestão da Atenção Primária à Saúde & $10(21,3)$ \\
\hline Gestão de Educação em Saúde & $15(31,9)$ \\
\hline $\begin{array}{l}\text { Coordenação Geral de Residência } \\
\text { Médica }\end{array}$ & $6(12,8)$ \\
\hline Outros & $8(17,0)$ \\
\hline \multicolumn{2}{|l|}{ Tempo de atuação como gestor $(\mathrm{n}=48)$} \\
\hline Até 1 ano & $12(25,0)$ \\
\hline $2-3$ anos & $18(37,5)$ \\
\hline $4-5$ anos & $8(16,7)$ \\
\hline 6 anos ou mais & $10(20,9)$ \\
\hline
\end{tabular}

Fonte: Elaborado pelos autores.

ativa, colaborando com a instituição de ensino no projeto pedagógico e no processo seletivo para ingresso dos médicos, definindo o número de vagas ofertadas e planejando a inserção nos diversos cenários de prática.
Em relação à consolidação dos PRMFC existentes, em sua maioria, os municípios não possuem planos ou estratégias específicas para (1) melhoria da infraestrutura dos serviços com PRMFC ( $n=30 ; 62,5 \%)$; para (2) organização dos processos de trabalho singularizados nos locais de funcionamento do PRMFC ( $\mathrm{n}=30 ; 62,5 \%)$, como definição de horário para estudos e agenda de atendimentos com modelagem pensada para o residente; para (3) avaliação das ações de integração ensino-serviço $(\mathrm{n}=31 ; 64,6 \%)$; para (4) atração de MFCs com incentivo financeiro $(n=25 ; 53,2 \%)$; e (5) para contratação dos MFCs egressos dos PRMFC do município ( $\mathrm{n}=25$; 52,1\%) (Tabela 2).

Em contrapartida, a maior parte dos gestores relatou a realização de concurso para MFC em sua gestão $(n=31 ; 64,6 \%)$, a disponibilização de bolsa complementar ao residente $(n=30 ; 62,5 \%)$ e a existência de programa de educação continuada voltada aos médicos $(\mathrm{n}=33 ; 68,8 \%)$, não sendo possível analisar as características de cada iniciativa em detalhe.

Em síntese, esses resultados mostram maior fragilidade dos municípios no planejamento do PRM e na melhoria da estrutura e dos processos de trabalho dos serviços com PRM, ao mesmo tempo em que sinalizam um esforço do gestor em incorporar e fixar o MFC em sua rede de atenção. Isso sugere que, na escassez de estratégias locais de gestão e fortalecimento da residência médica, muitos PRMFC provavelmente são incorporados e mesclados aos serviços de saúde em seu funcionamento cotidiano sem grandes adequações específicas para o processo de ensino-aprendizagem.

Historicamente a estratégia de complementação da bolsa do residente tem sido uma das principais iniciativas para se aumentar a ocupação de vagas de residência em MFC, com relativo êxito quando se comparam municípios que adotam ou não esta estratégia ${ }^{19,30}$. Entretanto, as estratégias de fixação desse especialista egresso da residência no âmbito dos serviços de APS dos municípios são raras.

Se por um lado o treinamento em serviço remunerado através de bolsa de estudos pode ser atrativo ao gestor municipal como uma economia de recursos no curto prazo, por outro dificilmente será suficiente para garantir que o médico egresso do PRMFC continue trabalhando no mesmo município, proporcionando maior longitudinalidade do cuidado. Sem uma estratégia sustentável de fixação dos profissionais egressos, investimentos na formação profissional podem 
Tabela 2. Estratégias relatadas por gestores municipais em saúde para o fortalecimento dos Programas de Residência Médica nos municípios, Brasil, 2020.

\begin{tabular}{|c|c|}
\hline Variável & n $(\%)$ \\
\hline \multicolumn{2}{|c|}{ Estratégias da gestão para fomentar a procura de graduandos pelas vagas nos PRM $(n=47)^{\star}$} \\
\hline Sim & $30(63,8)$ \\
\hline Não & $6(12,8)$ \\
\hline Não tem graduação no município & $10(21,3)$ \\
\hline Não sabe responder & $1(2,1)$ \\
\hline \multicolumn{2}{|c|}{ Plano de melhoria da infraestrutura de serviços com PRM ( $\mathrm{n}=48)$} \\
\hline $\operatorname{Sim}$ & $14(29,2)$ \\
\hline Não & $30(62,5)$ \\
\hline Não sabe responder & $4(8,3)$ \\
\hline \multicolumn{2}{|c|}{ Organização específica de processos de trabalho em serviços com PRM $(n=48)^{* *}$} \\
\hline Sim & $15(31,3)$ \\
\hline Não & $30(62,5)$ \\
\hline Não sabe responder & $3(6,3)$ \\
\hline \multicolumn{2}{|c|}{ Plano de avaliação das ações de integração ensino-serviço, incluindo RM ( $\mathrm{n}=48$ ) } \\
\hline $\operatorname{Sim}$ & $12(25,0)$ \\
\hline Não & $31(64,6)$ \\
\hline Não sabe responder & $5(10,4)$ \\
\hline \multicolumn{2}{|c|}{ Programa de educação continuada para qualificação dos médicos da APS ( $\mathrm{n}=48$ ) } \\
\hline Sim & $33(68,8)$ \\
\hline Não & $13(27,1)$ \\
\hline Não sabe responder & $2(4,2)$ \\
\hline \multicolumn{2}{|l|}{ Incentivo financeiro ao preceptor $(\mathrm{n}=48)$} \\
\hline Sim & $22(45,8)$ \\
\hline Não & $22(45,8)$ \\
\hline Não sabe responder & $4(8,3)$ \\
\hline \multicolumn{2}{|c|}{ Complementação do valor da bolsa do médico residente $(\mathrm{n}=48)$} \\
\hline Sim & $30(62,5)$ \\
\hline Não & $17(35,4)$ \\
\hline Não sabe responder & $1(2,1)$ \\
\hline \multicolumn{2}{|c|}{ Estratégia para contratação dos médicos egressos dos PRM locais $(\mathrm{n}=48)$} \\
\hline Sim & $21(43,8)$ \\
\hline Não & $25(52,1)$ \\
\hline Não sabe responder & $2(4,2)$ \\
\hline \multicolumn{2}{|l|}{ Realização de concurso público para MFC ( $\mathrm{n}=48)$} \\
\hline Sim & $31(64,6)$ \\
\hline Não & $17(35,4)$ \\
\hline Não sabe responder & $0(0,0)$ \\
\hline \multicolumn{2}{|c|}{ Incentivo financeiro específico para atração do especialista em MFC ( $\mathrm{n}=47)$} \\
\hline Sim & $20(42,6)$ \\
\hline Não & $25(53,2)$ \\
\hline Não sabe responder & $2(4,3)$ \\
\hline
\end{tabular}

PRM: Programa de Residência Médica; APS: Atenção Primária à Saúde; MFC: Medicina de Família e Comunidade. *Aproximação com a prática na APS durante a graduação; complementação com bolsa financeira; publicidade/divulgação; integração entre instituição de ensino e os serviços; valorização da função de preceptoria; qualificação da rede; integração do PRMFC com residência multidisciplinar. ${ }^{* *}$ Organização de agenda e horário para ensino-aprendizagem, adoção das normativas para ensino em serviço estabelecidas por órgãos governamentais.

Fonte: Elaborado pelos autores. 
ter seus benefícios limitados ao desenvolvimento de competências do indivíduo, com benefícios temporários para o sistema de saúde local.

Em relação à capacidade instalada no município para abertura de novas vagas de residência em MFC, a maior parte dos gestores respondeu que existe $(n=37 ; 77,1 \%)$, sendo que a maioria tem infraestrutura $(n=31 ; 83,3 \%)$, muitos têm recursos humanos $(n=23 ; 62,2 \%)$ e alguns têm recursos financeiros $(\mathrm{n}=12 ; 32,4 \%)$ para a ampliação das vagas. Ainda assim, 15 (31,2\%) gestores informaram que não pretendem realizar a abertura de novas vagas, trazendo como motivos a ausência de capacidade instalada, o baixo preenchimento das vagas já ofertadas, a ampliação recente do número de vagas e a dificuldade da compreensão de outros gestores sobre os serviços ofertados e sobre a residência em MFC.

$\mathrm{O}$ desenho desta pesquisa possui algumas fragilidades para analisar em profundidade a intencionalidade dos gestores em ter a RM em MFC como estratégia motriz de expansão da cobertura populacional de APS. Mesmo assim, esses achados apontam para uma boa margem para expansão da RM em MFC nos municípios com a infraestrutura local e recursos humanos já disponíveis. Contudo, a escassez de políticas de aporte de recursos financeiros para estruturação da APS e da RM em MFC e estímulo ao preenchimento das vagas de RM ofertadas pode estar limitando sobremaneira este movimento. Isto poderia, junto às políticas de provimento, levar cuidados primários de qualidade a maiores contingentes populacionais, com a ressalva de alcançar resultados de longo prazo mais satisfatórios por ter o potencial de articular reflexões sobre a qualidade e o modelo da atenção, à formação e qualificação profissional, a expansão da cobertura populacional, e a melhoria da estrutura dos serviços de saúde ${ }^{19,20}$.

\section{Efeitos da residência médica no sistema e serviços de saúde}

A maioria dos gestores $(n=32 ; 68,1 \%)$ relatou que a implantação de PRMFC no município impactou positivamente o sistema e serviços de saúde locais, fortalecendo os atributos da APS, em especial o acesso, maior qualidade e satisfação com o cuidado prestado à população, seja por trazer para mais próximo do serviço uma perspectiva de ensino-aprendizagem mais sistemática, seja por aumentar o aporte de recursos financeiros para a APS. Para um dos gestores entrevistados, o PRMFC:
Colabora com a organização e qualificação do acesso na APS; traz para o cenário de práticas a academia, estimulando a pesquisa, conhecimento e inovação; qualifica a atenção à saúde; colabora com a política de integração ensino serviço e comunidade, bem como com a gestão (Gestor 1).

Ao olhar dos gestores, o PRMFC torna-se um dispositivo que fortalece a atenção à saúde no serviço, modificando as formas como a clínica é exercida pelos profissionais, mas também potencializando as características de intervenção no território, essenciais na ESF. Os gestores apontam para um movimento de maior preocupação dos profissionais com as práticas desenvolvidas no cotidiano da equipe, com maior articulação com o território, e com produção de vínculos mais potentes:

[Há a] necessidade de qualificação da APS e competências dos médicos assistentes, [e o PRMFC contribui] habilitando, desenvolvendo a carteira de serviços da APS mas, principalmente, mudando a atitude assistencial, entre as quais, mas não somente: vigilância em saúde, clínica ampliada, humanização, promoção da participação popular ativa, qualificação da longitudinalidade da atenção e regulação da transferência do cuidado pelas equipes (Gestor 2).

Esta consolidação do modelo de atenção no serviço com PRMFC, na perspectiva dos gestores, gera resultados positivos importantes para o sistema de saúde municipal ao aumentar a resolutividade do cuidado a partir de equipes de saúde mais reflexivas e comprometidas com a qualidade da atenção; ao aumentar a diversidade de ações desenvolvidas pelos profissionais; e ao aumentar o número de atendimentos realizados pela equipe de saúde; o que consequentemente torna o usuário do serviço mais satisfeito:

O Programa [de RM] consegue transformar a assistência. Reduz solicitação de exames, encaminhamentos. Amplia a carteira de serviços da APS; Aumenta a satisfação do usuário e resolubilidade (Gestor 3).

[O PRM] potencializa o trabalho das equipes de saúde, não só em número de atendimentos, como também em iniciativas de promoção e prevenção da saúde (Gestor 4).

[O PRM] faz um trabalho excelente, de custo menor e maior garantia de fixação profissional (Gestor 5).

Além de agregar a interação ensino-serviço-comunidade [...], vislumbra-se no atual modelo de financiamento da Atenção Básica um maior aporte de recursos [para] os municípios com o programa de residência (Gestor 6 ). 
Outro efeito da implantação do PRMFC no município, relatado por cinco gestores, é a maior fixação do médico na equipe de saúde, o que, atrelado a sua maior qualificação e especialização, contribui para que a longitudinalidade do cuidado aconteça, fortalecendo a APS. Essa maior fixação na perspectiva dos gestores, se dá pelo fato de o médico residente permanecer por pelo menos dois anos no serviço; pelo maior investimento no médico preceptor e na equipe, criando um ambiente de trabalho mais potente; $\mathrm{e}$ pelo potencial de contratação do MFC egresso do PRMFC pelo município no futuro. Interessante notar que, destes cinco gestores, todos afirmaram na parte quantitativa que dispõem de mecanismos de contratação dos egressos dos PRMFC (por exemplo, abertura de concursos em data próxima à saída dos egressos do PRM, ajustes de carga horária ou garantia de vínculo ao mesmo serviço onde fez a RM) e três informaram que possuem incentivos financeiros específicos para MFCs, embora dois deles não tenham lançado concurso público em seu período de gestão, reforçando a tese de que o entendimento da potência do PRMFC para o sistema local pode não se desdobrar em práticas gerenciais de fomento em alguns lugares.

Estas ações podem levar municípios que as tenham adotado como política pública a um círculo virtuoso, impactando diretamente na raíz de problemas crônicos da APS e da saúde pública no Brasil: baixa qualificação dos profissionais de saúde, qualidade precária da atenção à saúde ofertada, baixo provimento e fixação de profissionais e expansão limitada de acesso dos serviços de APS ${ }^{19,20,30,31}$.

Estes achados somam-se à literatura no sentido de sugerirem que PRMFC podem se constituir numa estratégia que produz expansão da cobertura de APS a um custo favorável com claros benefícios de curto e longo prazo. Vislumbra-se assim uma política que pode contribuir na superação de problemas históricos da APS brasileira, como alta rotatividade de profissionais, vazios assistenciais em regiões de alta vulnerabilidade social, baixa qualidade do cuidado e insuficiência de especialistas em MFC $^{31-34}$.

\section{Efeitos da residência médica na formação profissional}

Embora tenha aparecido com menor frequência nas falas dos gestores, a capacitação profissional e formação médica continuada são descritas pelos entrevistados como um dos efei- tos positivos dos PRMFC. Isso ganha um sentido amplo desde a superação de problemas da formação médica, como também a potencialização da formação multiprofissional:

Os residentes motivam os demais profissionais das equipes, no sentido de ampliação do conhecimento, contribui na reorganização do processo de trabalho das equipes, qualifica o acolhimento dos usuários na unidade de saúde. Elabora projeto de intervenção na comunidade, considerando o perfil epidemiológico da população do território e por contribuir na formação destes profissionais para atuação no SUS (Gestor 7).

Empoderar o "SUS como escola", no dizer de um gestor, afeta não somente os médicos, mas todos os profissionais de saúde. Uma das questões mais importantes levantadas pelos gestores mostra que os processos de ensino-aprendizagem disparados e/ou potencializados pelo PRMFC motivam a equipe para outras formas de atuar e possibilitam a integração das múltiplas políticas de saúde que operam no serviço. Os gestores sugerem que o médico residente, em seu processo de ensino-aprendizagem, provoca e tensiona as práticas da equipe, o que permite que o PRMFC não se cristalize como uma política isolada no âmbito do serviço de saúde:

[...] o programa [de RM] estimula a educação continuada dos preceptores, colabora na integração da rede multiprofissional a partir do movimento de integração do programa de MFC com o programa Multiprofissional em Atenção Básica e atuação conjunta com os NASF. Os residentes tendem a provocar mais a discussão dos casos mais complexos com outros profissionais que possam colaborar. Residentes realizam produção científica (TCC) $e$ intervenções significativas na rede, algumas simples, mas de grande impacto. Ademais, residentes, especialmente do $2^{\circ}$ ano, tem potencial para realizar matriciamento junto aos demais profissionais da rede (Gestor 4).

Tudo isso faz com que este profissional em formação se aproxime das características desejadas para o médico que atua na APS. Para além de um profissional que atua em equipe de forma potente, que articula políticas e ações e que provoca a equipe para inovações na intervenção no território, o PRMFC possibilita a formação de um médico que atua de maneira mais abrangente e integral, adotando perspectivas mais complexas sobre o adoecimento e o estado de saúde da população.

[...] o programa de residência possibilita a formação de médicos com perfil direcionado à atenção básica, compreendendo todo o processo de saúde- 
doença do indivíduo, família e contexto social de inserção dos mesmos (Gestor 6).

Tais transformações na formação profissional potencializadas pelo PRMFC explicam em parte muitos dos resultados positivos observados em nível de sistema de saúde. E apesar de a abordagem quantitativa do estudo e as reflexões dos gestores trazerem a perspectiva de educação continuada de forma literal, é possível vislumbrar que o PRMFC pode abrir caminhos formativos mais complexos, mais interativos e participativos, mais ancorados no agir cotidiano dos profissionais diante da complexa realidade na qual se situam e operam os serviços. Ao potencializar movimentos analíticos no contexto das equipes, o PRMFC acaba por se constituir como um potencial dispositivo de educação permanente e não apenas de educação continuada como a priori pode transparecer ${ }^{35,36}$.

\section{Percepções dos gestores sobre o médico residente e o PRMFC}

Os resultados deste estudo também nos permitem identificar ao menos duas construções sobre o "ser" residente apresentadas pelos gestores em seus relatos. Os residentes motivam os profissionais, provocam discussões sobre os usuários atendidos e propõem e implementam intervenções que frequentemente não fazem parte do cotidiano da equipe. Com isso, conforme já afirmado anteriormente, ajudam no fortalecimento de princípios e modelos de atenção com resultados positivos para o sistema e o serviço.

Ao mesmo tempo, alguns gestores retratam o residente como o sujeito que vai aumentar o acesso aos atendimentos e a resolubilidade do cuidado. Possivelmente, o que emerge dessas narrativas dos gestores é a percepção dos residentes sob uma ótica de provimento e ocupação de espaços assistenciais de forma a aumentar a capacidade de produção do serviço. Percebe-se então a produção de um plano que parte de uma visão mais instrumental do médico residente, o que permite compreender, por exemplo, as motivações para um gestor ver este sujeito como aquele que vai "assumir uma equipe da ESF em casos de férias ou licença saúde/maternidade evitando o corte no repasse dos recursos" (Gestor 8).

Estes dois planos não constituem necessariamente polos positivo e negativo de uma relação de ensino-aprendizagem mais ampla e potente ou mais restrita e instrumental. Apesar de, na maioria dos relatos, estes planos identificados surgirem isolados, ambos também aparecem juntos em relatos de gestores, mostrando que o médico residente e o PRMFC podem e é desejável que promovam cuidado integral e aumentem a produção do serviço.

\section{Limitações e Potencialidades do estudo}

Este estudo apresenta algumas limitações. Primeiro, o número de gestores que participaram voluntariamente é relativamente baixo, embora participação de 30\% dos potenciais sujeitos em pesquisa com questionário online esteja dentro das estimativas de taxa de resposta expostas na literatura ${ }^{37}$. Segundo, o corpus textual para a análise qualitativa é oriunda de textos digitados pelos gestores nas questões abertas. Este é um procedimento de pesquisa válido, mas a não interação in loco com a pessoa limita a apreensão e compreensão do fenômeno em sua complexidade, deixando lacunas importantes. O questionário também não explorou o uso das diferentes políticas públicas federais como estratégias de fortalecimento dos serviços locais, não sendo possível compreender adequadamente a inserção do PRMFC no conjunto das políticas que incidem na APS municipal. Em contrapartida, o estudo tem o pioneirismo de explorar quais são os facilitadores e entraves para a formação de recursos humanos para a APS no SUS, através das RMFC.

\section{Considerações finais}

A RMFC é importante para o fortalecimento e a expansão da APS e para qualificação da rede local de serviços de saúde e consequente consolidação do SUS. O resultado deste estudo demonstra que apenas parte dos gestores municipais é capaz de identificar este potencial, porém nem sempre identificar significa colocar em prática políticas que fortaleçam essa potência. Soma-se a essa constatação o dado da literatura de que poucos municípios se destacaram no cenário nacional por utilizarem a residência médica como fator propulsor da APS. Chama a atenção que esse cenário aconteça mesmo após os esforços dos últimos 15 anos para implementação de políticas públicas que buscavam fortalecer os PRMFC.

A RMFC não pode ser considerada a única estratégia de expansão de cobertura populacional nos mais diferentes e heterogêneos contextos sociais do país. Mas a compreensão deste processo pode levar a gestão municipal a considerar esta possibilidade de intervenção em seu sistema de saúde, principalmente quando se tem revelado 
um caminho que pode levar a um círculo virtuoso significativo, como é o caso da RM.

Além do exposto, novas perguntas ainda precisam ser respondidas para o avanço na formação de recursos humanos para APS no SUS, por exemplo: Quais os entraves para a implementação plena das políticas federais? Por que apenas uma parcela dos gestores enxergam o potencial das residências médicas para o fortalecimento do sistema de saúde? Essas respostas são cruciais para a revisão das políticas e o ajuste de rotas para a expansão e fortalecimento dos PRMFC atrelado a maior ocupação de vagas dos programas já existentes.

Ao levantarmos evidências sobre a compreensão dos gestores e sobre sua atuação na relação ensino-serviço na APS, esperamos que seja possível dar maior visibilidade ao papel desses importantes atores na formação de recursos humanos para o SUS.

\section{Colaboradores}

Concepção e delineamento do estudo: APT Leite, AP Waquil, S Mai, VS Rosa, EAA Cordero e MEB Pinto. Análise dos dados: APT Leite, AP Waquil, TD Sarti, IB Correia, MEB Pinto, EAA Cordero, VS Rosa e S Mai. Interpretação dos dados: APT Leite, IB Correia, PS Chueiri, TD Sarti, AG Jantsch, AP Waquil, S Mai, VS Rosa, EAA Cordero, DK Augusto, AF Lopes e MEB Pinto. Redação do manuscrito inicial do artigo: APT Leite, IB Correia, PS Chueiri, TD Sarti e AG Jantsch. Revisão crítica do artigo: APT Leite, IB Correia, PS Chueiri, TD Sarti, AG Jantsch, AP Waquil, S Mai, VS Rosa, EAA Cordero, DK Augusto, AF Lopes, MEB Pinto. Todos os autores aprovaram a versão final deste artigo e concordam com os termos de copyright da revista. 


\section{Referências}

1. Sanders D, Nandi S, Labonté R, Vance C, Van Damme W. From primary health care to universal health coverage-one step forward and two steps back. Lancet 2019; 394(10199):619-621.

2. Hone T, Macinko J, Millett C. Revisiting Alma-Ata: what is the role of primary health care in achieving the Sustainable Development Goals? Lancet 2018; 392(10156):1461-1472.

3. Macinko J, Starfield B, Shi L. The contribution of primary care systems to health outcomes within Organization for Economic Cooperation and Development (OECD) countries, 1970-1998. Health Serv Res 2003; 38(3):831-865

4. Giovanella L, Franco CM, Almeida PF. Política Nacional de Atenção Básica: para onde vamos? Cien Saude Colet 2020; 25(4):1475-1482.

5. Starfield B, Shi L, Macinko J. Contribution of primary care to health systems and health. Milbank Q. 2005; 83(3):457-502.

6. Brasil. Ministério da Saúde (MS). Portaria Interministerial $n^{\circ} 1.802$, de 26 de agosto de 2008 Institui o Programa de Educação pelo Trabalho para a Saúde - PET - Saúde. Diário Oficial da União 2008; 26 ago.

7. Brasil. Casa Civil. Decreto no 7.385, de 8 de dezembro de 2010. Institui o Sistema Universidade Aberta do Sistema Único de Saúde - UNA-SUS, e dá outras providências. Diário Oficial da União 2010; 8 dez.

8. Brasil. Ministério da Educação (MEC). Ministério da Saúde (MS). Portaria Interministerial $\mathrm{n}^{\circ} 1.001$, de 22 de outubro de 2009. Institui o Programa Nacional de Apoio à Formação de Médicos Especialistas em Áreas Estratégicas - PRÓ-RESIDÊNCIA. Diário Oficial da União 2009; 22 out.

9. Brasil. Ministério da Saúde (MS). Portaria Interministerial $\mathrm{n}^{\circ}$ 2.087, de 1 de setembro de 2011. Institui o Programa de Valorização do Profissional da Atenção Básica. Diário Oficial da União 2011; 1 set.

10. Brasil. Lei $\mathrm{n}^{\circ} 12.871$, de 22 de outubro de 2013. Institui o Programa Mais Médicos, altera as Leis $n^{\circ} 8.745$, de 9 de dezembro de 1993, e $\mathrm{n}^{\circ} 6.932$, de 7 de julho de 1981, e dá outras providências. Diário Oficial da União 2013; 23 out.

11. Brasil. Lei ${ }^{\circ} 13.958$, de 18 de dezembro de 2019, Institui o Programa Médicos pelo Brasil, no âmbito da atenção primária à saúde no Sistema Único de Saúde (SUS), e autoriza o Poder Executivo federal a instituir serviço social autônomo denominado Agência para o Desenvolvimento da Atenção Primária à Saúde (Adaps). Diário Oficial da União 2019; $19 \mathrm{dez}$.

12. Oliveira APC, Gabriel M, Poz MRD, Dussault G. Desafios para assegurar a disponibilidade e acessibilidade à assistência médica no Sistema Único de Saúde. Cien Saude Colet 2017; 22(4):1165-1180.

13. Oliveira BLCA, Lima SF, Pereira MUL, Pereira Júnior GA. Evolução, distribuição e expansão dos cursos de medicina no Brasil (1808-2018). Trab Educ Saude 2019; 17(1):e0018317.

14. Scheffer M, coordenador. Demografia Médica no Brasil 2020. São Paulo: Departamento de Medicina Preventiva da Faculdade de Medicina da USP, Conselho Federal de Medicina; 2020.
15. Rodrigues PHA, Ney MS, Paiva CHA, Souza LMBM. Regulação do trabalho médico no Brasil: impactos na Estratégia Saúde da Família. Physis 2013; 23(4):11471166.

16. van der Leeuw RM, Lombarts KM, Arah OA, Heineman MJ. A systematic review of the effects of residency training on patient outcomes. BMC Med 2012 10(1):65.

17. Simas KBF, Gomes AP, Simões PP, Augusto DK, Siqueira-Batista R. A residência de Medicina de Família e Comunidade no Brasil: Breve recorte histórico. Rev Bras Med Fam Comunidade 2018; 13(40):1-13.

18. Augusto DK, David L, Oliveira DOPS, Trindade TG, Lermen Junior N, Poli Neto P. Quantos médicos de família e comunidade temos no Brasil? Rev Bras Med Fam Comunidade 2018; 13(40):1-4.

19. Storti MMT, Oliveira FP, Xavier AL, Storti MMT, Oliveira FP, Xavier AL. A expansão de vagas de residência de Medicina de Família e Comunidade por municípios e o Programa Mais Médicos. Interface (Botucatu) 2017; 21(Supl. 1):1301-1314.

20. Soares RS, Oliveira FP, Neto AJM, Barreto DS, Carvalho ALB, Sampaio J, Figueiredo AM. Residência em medicina de família e comunidade: construindo redes de aprendizagens no SUS. Rev Bras Med Fam Comunidade 2018; 13(40):1-8.

21. Sarti TD, Fontenelle LF, Gusso GDF. Panorama da expansão dos programas de Residência Médica em Medicina de Família e Comunidade no Brasil: desafios para sua consolidação. Bras Med Fam Comunidade $2018 ; 13(40): 1-5$.

22. World Health Organization (WHO). Global Strategy on Human Resources for Health: Workforce 2030. Genebra: WHO; 2016.

23. Sales M, Kieny MP, Krech R, Etienne C. Human resources for universal health coverage: from evidence to policy and action. Bull World Health Organ 2013; 91(11):798A.

24. Oliveira FP, Vanni T, Pinto HA, Santos JTR, Figueiredo AM, Araújo SQ, Matos MFM, Cyrino EG. Mais Médicos: um Programa brasileiro em perspectiva internacional. Interface (Botucatu) 2015; 19(54):623634.

25. Minayo MCS. O desafio do conhecimento: pesquisa qualitativa em saúde. 14ª ed. São Paulo: Hucitec; 2014.

26. Ouverney ALM, Carvalho ALB, Machado NMS, Moreira MR, Ribeiro JM. Gestores municipais do Sistema Único de Saúde: perfil e perspectivas para o Ciclo de Gestão 2017-2020. Saude Debate 2019; 43(esp. 7):7591.

27. Brasil. Ministério da Saúde (MS). Cadastro Nacional dos Estabelecimentos de Saúde do Brasil (CNES) [Internet]. [acessado 2020 out 23]. Disponível em: http://tabnet.datasus.gov.br/cgi/deftohtm.exe?cnes/ cnv/estabbr.def.

28. Brasil. Ministério da Saúde (MS). Direm - Diretório das Escolas de Medicina [Internet]. [acessado 2020 out 23]. Disponível em: https://direm.org/.

29. Rodrigues ET, Forster AC, Santos LL, Ferreira JBB, Falk JW, Fabbro ALD. Perfil e trajetória profissional dos egressos da Residência em Medicina de Família e Comunidade do Estado de São Paulo. Rev Bras Educ Med 2017; 41(4):604-614. 
30. Barreto DS, Melo Neto AJ, Figueiredo AM, Sampaio J, Gomes LB, Soares RS. The More Doctors Program and Family and Community Medicine residencies: articulated strategies of expansion and interiorization of medical education. Interface (Botucatu) 2019; 23(Supl. 1):e180032.

31. Justino ALA, Oliver LL, Melo TP. Implantação do programa de residência em medicina de família e comunidade da secretaria municipal de saúde do rio de janeiro, Brasil. Cien Saude Colet 2016; 21(5):1471-1480.

32. Pinto LF, Giovanella L. Do Programa à Estratégia Saúde da Família: expansão do acesso e redução das internações por condições sensíveis à atenção básica (Icsab). Cien Saude Colet 2018; 23(6):1903-1914.

33. Andrade LR, Pinto ICM, Soares CLM, Silva VO. Provimento e fixação de médicos na atenção primária à saúde no estado da Bahia. Rev Admin Publica 2019; 53(3):505-519.

34. Ney MS, Rodrigues PHA. Fatores críticos para a fixação do médico na Estratégia Saúde da Família. Physis 2012; 22(4):1293-1311.

35. Ceccim RB. Educação permanente em saúde: desafio ambicioso e necessário. Interface (Botucatu) 2005; 9(16):161-177.

36. Arnemann CT. Educação permanente em saúde no contexto da residência multiprofissional: estudo apreciativo crítico [tese]. Porto Alegre: Universidade Federal do Rio Grande do Sul; 2017.

37. Wachelke J, Natividade J, Andrade A, Wolter R, Camargo B. Caracterização e avaliação de um procedimento de coleta de dados online (Corp). Avaliaç Psicol 2014; 13(1):143-146.

Artigo apresentado em 23/10/2020

Aprovado em 10/02/2021

Versão final apresentada em 12/02/2021

Editores-chefes: Maria Cecília de Souza Minayo, Romeu

Gomes, Antônio Augusto Moura da Silva 